University of Wollongong

Research Online

Australian Institute for Innovative Materials -

Papers

Australian Institute for Innovative Materials

$1-1-2013$

\title{
A significant improvement in both low- and high-field performance of MgB2 superconductors through graphene oxide doping
}

K S B De Silva

University of Wollongong, kaludewa@uow.edu.au

S H Aboutalebi

University of Wollongong, sha942@uowmail.edu.au

Xun Xu

University of Wollongong, xun@uow.edu.au

Xiaolin Wang

University of Wollongong, xiaolin@uow.edu.au

W X Li

University of Wollongong, wenxian@uow.edu.au

See next page for additional authors

Follow this and additional works at: https://ro.uow.edu.au/aiimpapers

Part of the Engineering Commons, and the Physical Sciences and Mathematics Commons

Research Online is the open access institutional repository for the University of Wollongong. For further information contact the UOW Library: research-pubs@uow.edu.au 


\title{
A significant improvement in both low- and high-field performance of MgB2 superconductors through graphene oxide doping
}

\author{
Abstract \\ The effects of graphene oxide (GO) doping on the superconducting properties of MgB2 were studied \\ using bulk samples made by the diffusion method. Homogeneous dispersions of $\mathrm{GO}$ in tetrahydrofuran \\ were obtained through a novel synthesis method, which is then chemically doped with MgB2. It was found \\ that GO doping significantly improves the critical current density, under both low and high magnetic fields, \\ which distinguishes GO from all the other elements doped into MgB2 so far. 2013 Acta Materialia Inc. \\ Published by Elsevier Ltd. All rights reserved.
}

\section{Keywords}

improvement, both, low, high, field, significant, performance, doping, mgb2, superconductors, graphene, oxide

Disciplines

Engineering | Physical Sciences and Mathematics

\section{Publication Details}

De Silva, K., Aboutalebi, S., Xu, X., Wang, X., Li, W., Konstantinov, K. \& Dou, S. X. (2013). A significant improvement in both low- and high-field performance of MgB2 superconductors through graphene oxide doping. Scripta Materialia, 69 (6), 437-440.

\section{Authors}

K S B De Silva, S H Aboutalebi, Xun Xu, Xiaolin Wang, W X Li, Konstantin Konstantinov, and S X. Dou 


\title{
A significant improvement in both low and high field performance of $\mathrm{MgB}_{2}$ superconductors through graphene oxide \\ doping
}

\author{
K S B De Silva, S H Aboutalebi, X Xu, X L Wang, W X Li, K Konstantinov and S X \\ Dou*
}

Institute for Superconducting and Electronic Materials, University of Wollongong, Northfields Avenue, Wollongong, New South Wales, 2522, Australia, Fax:61-2-4221 573; Tel: 61-2-4221 4558, E-mail: shi_dou@uow.edu.au

\begin{abstract}
The effects of graphene oxide (GO) doping on the superconducting properties of $\mathrm{MgB}_{2}$ were studied using bulk samples made by the diffusion method. Homogeneous dispersions of GO in tetrahydrofuran (THF) were obtained through a novel synthesis method, which is then chemically doped with $\mathrm{MgB}_{2}$. It was found that GO doping significantly improves the critical current density, both at low and at high magnetic fields, which distinguishes GO from all the other elements doped into $\mathrm{MgB}_{2}$ so far.
\end{abstract}

Key words: Superconductivity, electrical properties, GO-doping; Diffusion

Since the discovery of superconductivity in $\mathrm{MgB}_{2}$ [1], extensive research has been undertaken to enhance its superconducting properties, such as critical current density $\left(\mathrm{J}_{\mathrm{c}}\right)$, upper critical field $\left(\mathrm{H}_{\mathrm{c} 2}\right)$, and irreversibility field $\left(\mathrm{H}_{\mathrm{irr}}\right)$. Many studies have shown that, carbon containing dopants, including silicon carbide (SiC), carbon nanotubes (CNTs), hydrocarbons/carbohydrates, graphite, and graphene are effective means to enhance the field 
dependence of $\mathrm{J}_{\mathrm{c}}$ and $\mathrm{H}_{\mathrm{c} 2}$ of $\mathrm{MgB}_{2}$. Most carbon dopants, however, adversely affect $\mathrm{T}_{\mathrm{c}}$ and the low field $\mathrm{J}_{\mathrm{c}}$ performance [2-7].

Among these dopants, graphene has been recognized as a relatively new dopant for $\mathrm{MgB}_{2}$ due to its specific way of improving $\mathrm{J}_{\mathrm{c}}$. It improves the intergrain connectivity, and at the same time, leaves micro-strains in the $\mathrm{MgB}_{2}$ matrix which are beneficial for improving the flux pinning [6]. Although, the high cost of pure graphene renders it impractical for large-scale applications. Furthermore, the re-stacking of graphene sheets during the process results in an inevitable performance penalty. An easier way is to employ graphene oxide, which is typically monolayer in nature. It is used to make the composite material, and the final structure is then thermally reduced to prevent the aggregation of graphene sheets [8]. Apart from the facile synthesis method, graphene oxide has the advantages of cost and suitability for mass production, which make it sensible candidate for industrial scale applications. Since the agglomeration of additives has been always the main problem the solid state mixing, it is a great challenge to achieve a homogeneous dispersion of the nano-dopants in the matrix material. Although available well-dispersed aqueous colloids of exfoliated GO allow homogeneous mixing of dopant in $\mathrm{MgB}_{2}$, these aqueous solutions degrade the performance of $\mathrm{MgB}_{2}$. Tetrahydrofuran (THF) has been used as an organic solvent to disperse GO sheets, as it can satisfy all of the requirements of a suitable organic solvent that can prevent the oxidation of boron or Mg powder [9], while simultaneously dispersing GO. Dispersing GO in THF, however, is not regarded a straightforward task as it typically involves agglomeration of GO particles [10]. However, employing extremely large GO sheets in our case not only led to effective dispersion of GO sheets in THF, but also resulted in achieving graphene oxide liquid crystals in the THF. In this paper, we demonstrate that processibility of GO dispersions in organic solvents can be further exploited to fabricate doped $\mathrm{MgB}_{2}$ samples which can 
satisfy all of the above-mentioned requirements and exhibit extremely good low- and highfield performance.

The experimental set-up and procedure for the synthesis of graphene oxide (GO) were based on our previously reported synthesis methods $[11,12]$. (see supplementary information for details). GO-doped bulk samples were prepared based on our previously reported synthesis methods, using crystalline boron powder (0.2 to $2.4 \mu \mathrm{m} 99.999 \%), \mathrm{Mg}$ powder (99\%, 352 mesh), and a homogeneous suspension of GO, as precursors [7]. (see supplementary information for details)

A JEOL JSM-7500FA field emission scanning electron microscope (FESEM), equipped with an Ultra Thin Window (UTW) JEOL Hyper-Minicup energy dispersive spectrometer (EDS) was used for SEM analysis. The GO samples were investigated using an Asylum research MFP-3D Atomic Force Microscope (AFM). Images were collected using AC (tapping) mode imaging under ambient conditions with Mmasch NSC15 tips $\mathrm{Si}_{3} \mathrm{~N}_{4}$ coated silicon cantilevers. Transmission electron microscopy (TEM) was performed on GO powder sample using a JEOL $2011200 \mathrm{keV}$ analytical instrument. The phase identification and crystal structure investigations were carried out at room temperature using an X-ray diffractometer (GBCMMA) with $\mathrm{Cu}-\mathrm{K} \alpha$ radiation $(\lambda=1.54059 \AA)$. The Raman scattering was measured using a confocal laser Raman spectrometer (HORIBA Jobin Yvon system) with a 100x microscope. The $632.8 \mathrm{~nm}$ line of a HeNe laser with power of about $20 \mathrm{~mW}$ was used for excitation.

The superconducting transition temperature $\left(\mathrm{T}_{\mathrm{c}}\right)$ was determined from the AC susceptibility measurements, and the magnetic $\mathrm{J}_{\mathrm{c}}$ was derived from the width of the magnetization loop using Bean's model [13], using a Quantum Design physical properties measurement system (PPMS). The formula used is,

$J_{c}=20 \Delta M / a(1-a / 3 b)$ 
Here, $\Delta \mathrm{M}$ is the width of the relevant $\mathrm{M}(\mathrm{H})$ loop, and $a$ and $b$ are the width and length of the bar shaped sample, respectively, in a plane perpendicular to the applied field, with $a<b$. The resistivity measurements were conducted using the standard dc four-probe technique under magnetic fields up to $13 \mathrm{~T}$. The active cross-section $\left(\mathrm{A}_{\mathrm{F}}\right)$ was calculated from the resistivity, $\rho$, from Rowell's model [14].

The as-prepared GO samples were analyzed by SEM, AFM, and TEM investigations. AFM studies in conjunction with electron microscopy confirmed the monolayer nature of the asprepared GO dispersions. Our as-prepared GO dispersions mostly consisted of monolayer GO sheets with sheet sizes of approximately $50 \mu \mathrm{m}$, as evidenced by SEM and AFM (figure 1). In figure 1(a), the light black part is monolayer GO sample. The Black part shows the inevitable folded parts of one GO monolayer. The white part in SEM image is the substrate material which is Si. In figure 1(b) the step height measurement shows that the thickness of our as-prepared GO samples is around $0.87 \mathrm{~nm}$ which corresponds well with the reported literature data (thickness of monolayer GO is around $0.8-1.2 \mathrm{~nm}$ ). In the case of multi-layer GO sheets, the number of edge lines is a sign of the number of layers. However, in the current study as it is illustrated in figure 1(c), just one edge line can be observed in GO sample indicating that GO sheets are monolayer in nature. The polarized optical micrograph presented in figure $1(\mathrm{~d})$ reveals the nematic liquid crystalline nature of our as-prepared dispersions. The typical birefringence behaviour revealed through imaging between crossed polarisers confirmed that the dispersions exhibited the typical birefringence behaviour of liquid crystals [11]. Large-area graphene sheets are highly desirable, mainly because of the much lower amount of defects on their basal planes, arising from the lower amount of nonstoichiometric oxygen, which also makes the dispersion of GO in THF possible.

The XRD measurements were performed on the ground $\mathrm{MgB}_{2}$ pellets. According to the XRD patterns presented in figure 2(a), all peaks can be indexed to $\mathrm{MgB}_{2}$ lines corresponding to the 
hexagonal P6/mmm structure. The lattice parameters $a$ and $c$, the full width at half maximum (FWHM) of the peak (110), the grain size and the strain, are shown in table 1.

The refinement results indicate that both the $a$-axis and the $c$-axis parameters vary with increasing GO doping level. Increases in the $c / a$ ratio and a considerable shift in the (110) peak to higher angles with increasing doping level, indicate that some amount of carbon substitution has occurred in the B sites [15]. Among the various XRD peaks, the FWHM of the (110) peak is related to the in-plane crystallinity, while the FWHM of the (002) peak reflects the out-of-plane disorder [16]. According to the Williamson-Hall, the strain and the grain size affect the FWHM value [17]. The increased trend of the FWHM values obtained from the GO-doped samples compared to the undoped sample give evidence of reduced grain size and increased lattice strain due to doping. In fact, the calculated results on grain size and lattice strain from a Williamson-Hall plot like the experimental results given in table 1, which show a similar trend, would strengthen the above evidences even more. An increased trend towards more $\mathrm{MgO}$ is also observed with increasing the doping level.

Figure 2(b) shows the room temperature Raman spectra for the undoped, 1 wt. $\%$ and 2 wt. $\%$ GO-doped bulk samples in the range of 450 to $950 \mathrm{~cm}^{-1}$. There are two peaks visible in the measured range centred at about $610 \mathrm{~cm}^{-1}$ and $785 \mathrm{~cm}^{-1}$, similar to those reported in the literature. The low frequency Raman band is assigned to the Raman active $\mathrm{E}_{2 \mathrm{~g}}$ mode which is strongly coupled to the electronic conduction $\sigma$-band, while the high frequency band is attributed to the phonon density of states (PDOS) due to disorder [18]. Since doping affects the electron-phonon coupling intensity and crystal distortion, this will influence the Raman shift and the line width (FWHM) of the Raman scattering. As evidenced by figure 2(b), both the $E_{2 g}$ peak and PDOS peak of the GO-doped sample have slightly shifted to higher frequency, indicating that the band structure of the $\mathrm{MgB}_{2}$ shows a higher phonon vibration frequency due to carbon substitution at boron sites and increased lattice defects as a result of 
doping. Furthermore, the intensity of the PDOS distortion has become more pronounced in the doped sample, which implies increased disorder compared to the undoped sample [19].

Table 1 also presents the critical temperature, resistivity at $300 \mathrm{~K}$ and $40 \mathrm{~K}$, the residual resistivity ratio $\left(R R R=\rho_{300 K} / \rho_{40 K}\right)$, and the active cross-sectional area $\left(A_{F}\right)$ of the undoped and GO-doped $\mathrm{MgB}_{2}$ bulk samples. Instead of showing increased resistivity owing to increased inter-band and intra-band scattering in the $\sigma$-bands [14], the GO-doped samples exhibit low resistivities. This behaviour can be explained by the counteracting effects of improved connectivity and increased disorder due to GO doping. The stable dispersion of GO (figure 1) has resulted in a homogeneous dispersion of the dopant in the $\mathrm{MgB}_{2}$ matrix, which has effectively improved the inter-grain connectivity due to the absence of agglomerations of dopant. With increasing doping level, however, the resistivity tends to rise, owing to increased band scattering due to $C$ substitution. The increased $A_{F}$ values (table 1) reveal that the connectivity has been improved due to GO doping. The RRR, i.e., the ratio of the resistivity at $300 \mathrm{~K}$ to that at $40 \mathrm{~K}$, reflects the degree of disorder, where higher electron scattering leads to a low RRR value [20]. The fact that the RRR values for the GO-doped samples are smaller than that of the undoped sample again proves the increased disorder, which is in agreement with the results revealed in XRD and Raman analysis. It should be mentioned that GO doping appears to have another benefit, as it does not greatly affect on $\mathrm{T}_{\mathrm{c}}$. The in-field $\mathrm{J}_{\mathrm{c}}$ performances of the undoped and GO-doped $\mathrm{MgB}_{2}$ bulk samples as a function of applied magnetic field are shown in figure 3 . The $\mathrm{J}_{\mathrm{c}}$ performances of all doped samples have improved significantly under both low and high fields, showing considerably better results compared to the performances with other carbon dopants and doping levels. The sample with $1 \mathrm{wt} \% \mathrm{GO}$ showed a $\mathrm{J}_{\mathrm{c}}$ that was 27 times higher compared to that of the undoped sample at $5 \mathrm{~K}, 8 \mathrm{~T}$, with a slight drop in $\mathrm{T}_{\mathrm{c}}$ of just $1.2 \mathrm{~K}$. At the same time, it showed a $\mathrm{J}_{\mathrm{c}}$ of $6.35 \times 10^{5} \mathrm{~A} / \mathrm{cm}^{2}$ at $20 \mathrm{~K}$ at zero field, which was increased by a factor of 1.5 compared to 
the undoped $\mathrm{J}_{\mathrm{c}}$ value. This is very significant, as most carbon dopants tend to doping reduce $\mathrm{J}_{\mathrm{c}}$ at low fields. Although there is a continuous improvement in $\mathrm{J}_{\mathrm{c}}$ at high fields with increasing doping level, low-field $\mathrm{J}_{\mathrm{c}}$ starts to show a slight reduction when the doping level exceeds $1 \mathrm{wt} \%$. According to Matsushita et al. [21], a strong correlation exists between the connectivity and the self-field $\mathrm{J}_{\mathrm{c}}$. Therefore, better electrical connectivity, which means higher $A_{F}$ values, leads to higher self-field $J_{c}$ values. As can be seen from table 1, the doping level of $1 \mathrm{wt} \%$ shows the highest $A_{F}$ value, which is therefore consistent with the low-field $J_{c}$ improvement. The high-field $\mathrm{J}_{\mathrm{c}}$ performance, however, is greatly affected by $\mathrm{H}_{\mathrm{c} 2}$, and the flux pinning mechanism hence improves with increasing doping level. Furthermore, an increased amount of $\mathrm{MgO}$ was detected with increasing GO doping level through XRD analysis. These $\mathrm{MgO}$ precipitates also act as effective pinning centres, which would therefore improve the high field performance [22]. Moreover, increases in $\mathrm{H}_{\text {irr }}$, the FWHM value of the (110) peak, and strain, as well as decreased grain size indicate enhanced grain boundary pinning due to GO doping.

We have systematically studied the effects of GO doping on the microstructure, crystallinity, resistivity, and critical current density of $\mathrm{MgB}_{2}$ bulk samples. Increased lattice strain and disorder were observed due to the carbon substitution effect. Substantially enhanced $\mathrm{J}_{\mathrm{c}}$ properties under high fields and improved critical fields were observed in the GO doped samples with only a slight depression of $T_{c}$. A remarkable enhancement of the zero-field $J_{c}$ was obtained for the $1 \mathrm{wt} \%$ GO doped sample, owing to improved intergrain connectivity. This study will open up new opportunities for large-scale manufacturing of nano-doped $\mathrm{MgB}_{2}$ through a solution route with a cheap additive - GO, which is capable of improving both low and high field $\mathrm{J}_{\mathrm{c}}$. 


\section{Acknowledgements}

This research was performed with the support of the Australian Research Council (ARC) through Projects LP0989352, DP 1093952 and Hyper Tech Research Inc., OH, USA. The authors would like to thank Dr. T. Silver, I. Nevirkovets for their helpful discussions.

\section{References}

[1] J. Nagamatsu, N. Nakagawa, T. Muranaka, Y. Zenitani, J. Akimitsu, Nature 410 (2001) 63.

[2] J. H. Kim, S. Oh, Y-U. Heo, S. Hata, H. Kumakura, A. Matsumoto, M. Mitsuhara, S.

Choi, Y. Shimada, M. Maeda, J. L.MacManus-Driscoll, and S. X Dou. NPG Asia Mater 4 (2012) e3.

[3] S. X. Dou, W. Yeoh, J. Horvat, M. Ionescu Appl. Phys. Lett. 83 (2003) 4996

[4] S. X Dou., S.Soltanian, J.Horvat, X. L.Wang, S. H.Zhou, M.Ionescu, H. K.Liu, P.Munroe, T M.omsic, Appl. Phys. Lett. 81 (2002) 3419.

[5] C. Shekhar, R. Giri, R. S. Tiwari, O. N. Srivastava, S. K. Malik, J. Appl. Phys. 102 (2007) 093910.

[6] X. Xu, S. X. Dou, X. L. Wang, J. H. Kim, M. Choucair, W. K. Yeoh, R. K. Zheng S. P. Ringer, Supercond. Sci. Technol. 23 (2010) 085003.

[7] K. S. B.De Silva, S.Gambhir, X. L.Wang, X. Xu, W. X.Li, D. L.Officer, D.Wexler, G. G.Wallace, S. X.Dou J. Mat. Chem. 22 ( 2012) 13941.

[8] Tang H., Ehlert G. J., Lin Y., Sodano H. A. Nano Lett. 12 (2011) 84

[9] K -J.Jeon, H. R.Moon, A. M.Ruminski, B.Jiang, C. Kisielowski, R.Bardhan, J. J. Urban Nat. Mater. 10 (2011) 286.

[10] J. I.Paredes, S.Villar-Rodil, A.Martínez-Alonso, J. M .D.Tascón Langmuir 24 (2008) 10560. 
[11] S. H.Aboutalebi, M. M.Gudarzi, Q. B.Zheng, J -K.KimAdv. Funct. Mat. 21 (2011) 2978.

[12] S. H.Aboutalebi, A. T.Chidembo, M.Salari, K.Konstantinov, D.Wexler, H. K. Liu, S. X. Dou, Energy \& Environ. Sci. 4 (2011) 1855.

[13] C. P. Bean, Rev. Mod. Phys. 36 (1964) 31.

[14] J. M. Rowell, Supercond. Sci. Technol. 16 (2003) R17.

[15] M. Avdeev, J. D. Jorgensen, R. A.Ribeiro, S. L.Bud'ko, P. C Canfield, Physica C 387 (2003) 301.

[16] A.Yamamoto, J. Shimoyama, S.Ueda, Y.Katsura, I. Iwayama, S. Horii, K. Kishio, Appl. Phys. Lett. 86 (2005) 212502.

[17] G. K. Williamson, W. H.Hall Acta Metall. 1 (1953) 22.

[18] W. X. Li, Y. Li, R. H. Chen, R. Zeng, S. X. Dou, M. Y. Zhu, H. M. Jin, Phys. Rev. B 77 (2008) 094517.

[19] W. X.Li, R. H.Chen, Y.Li, M. Y. Zhu, H. M. Jin, R. Zeng, S. X. Dou , B. Lu, J.Appl. Phys. 103 (2008) 013511.

[20] X. L. Wang, S. X. Dou, M. S. A. Hossain, Z. X. Cheng, X. Z. Liao, S. R. Ghorbani, Q. W. Yao, J. H. Kim, T. Silver, Phys. Rev. B 81 (2010) 224514.

[21] M. Teruo, K. Masaru, Y.Akiyasu, S. Jun-ichi, K. S. Kohji, Supercond. Sci. Technol. 21 (2008) 015008.

[22] R. K Singh., Y.Shen, R.Gandikota, C.Carvalho, J. M.Rowell, N.Newman, Appl. Phys. Lett. 93 (2008) 242504. 
Table 1: Lattice constants $a, c$, full width at half maximum (FWHM), grain size, strain, Critical temperature $\left(\mathrm{T}_{\mathrm{c}}\right)$, resistivity at $300 \mathrm{~K}$ and $40 \mathrm{~K}$, residual resistivity ratio (RRR), and active cross-sectional area $\left(\mathrm{A}_{\mathrm{F}}\right)$ of undoped and GOdoped $\mathrm{MgB}_{2}$ bulk samples.

\begin{tabular}{|c|c|c|c|c|c|c|c|c|c|c|}
\hline \multirow[t]{2}{*}{ Sample } & \multicolumn{2}{|c|}{ Lattice constants } & \multirow{2}{*}{$\begin{array}{c}\text { FWHM } \\
\text { of (110) } \\
\left({ }^{\circ}\right)\end{array}$} & \multirow{2}{*}{$\begin{array}{l}\text { Grain } \\
\text { size } \\
(\mathrm{nm}) \\
\end{array}$} & \multirow{2}{*}{$\begin{array}{r}\text { Strain } \\
(\%) \\
\end{array}$} & \multirow[t]{2}{*}{$\mathrm{T}_{\mathrm{c}}$} & \multirow{2}{*}{$\begin{array}{r}\rho(300 \mathrm{~K}) \\
(\mu \Omega \mathrm{cm})\end{array}$} & \multirow{2}{*}{$\begin{array}{l}\rho(40 \mathrm{~K}) \\
(\mu \Omega \mathrm{cm})\end{array}$} & \multirow[t]{2}{*}{ RRR } & \multirow[t]{2}{*}{$\mathrm{A}_{\mathrm{F}}$} \\
\hline & $\mathrm{a}(\AA)$ & $c(\AA)$ & & & & & & & & \\
\hline undoped & 3.0884 & 3.526 & 0.3420 & 195.7 & 0.1336 & 38.9 & 47.02 & 12.74 & 3.69 & 0.125 \\
\hline $0.5 \%$ GO doped & 3.0875 & 3.5307 & 0.4000 & 167.4 & 0.2025 & 38.1 & 33.62 & 11.85 & 2.83 & 0.197 \\
\hline $1.0 \%$ GO doped & 3.0869 & 3.5316 & 0.4200 & 159.7 & 0.2045 & 37.7 & 21.4 & 9.08 & 2.35 & 0.349 \\
\hline $1.5 \%$ GO doped & 3.084 & 3.5283 & 0.4580 & 128.9 & 0.2038 & 37.3 & 28.39 & 12.93 & 2.19 & 0.278 \\
\hline
\end{tabular}

\section{Figure captions :}

Figure 1. a) SEM, b) AFM, c) high resolution TEM micrographs of as-prepared GO sheets and d) polarized optical micrograph of LC graphene oxide dispersion inTHF.

Figure 2.(a) XRD patterns for undoped and GO- doped $\mathrm{MgB}_{2}$.(b) Raman spectra with Gaussian fitted $\mathrm{E}_{2 \mathrm{~g}}$ mode and PDOS distortion of undoped GO-doped $\mathrm{MgB}_{2}$.

Figure 3. In-field $\mathrm{J}_{\mathrm{c}}$ performance of undoped, and GO-doped $\mathrm{MgB}_{2}$ bulk samples. Inset shows an enlarged view of the low-field $\mathrm{J}_{\mathrm{c}}$ at $20 \mathrm{~K}$ 

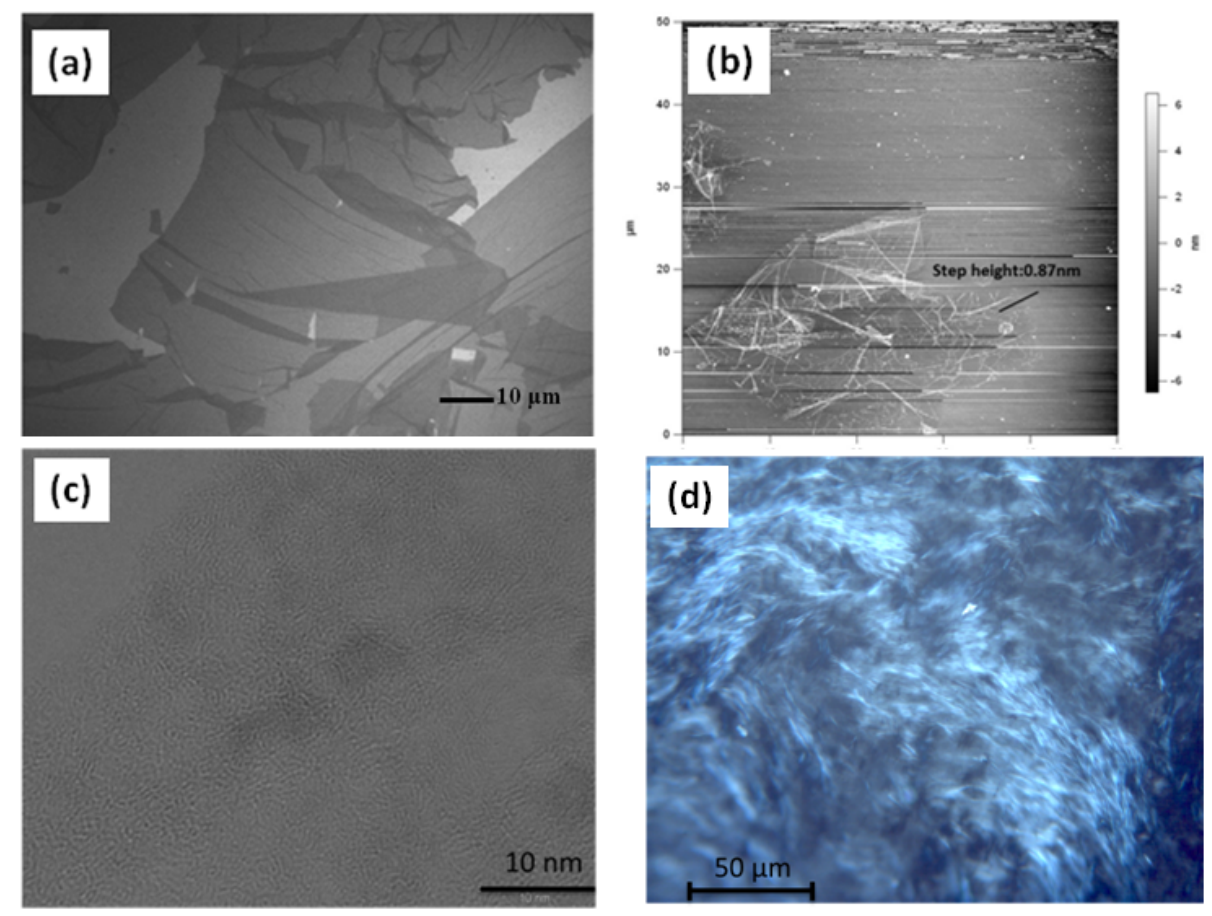

Figure 1. a) SEM, b) AFM, c) high resolution TEM micrographs of as-prepared GO sheets and d) polarized optical micrograph of LC graphene oxide dispersion inTHF.
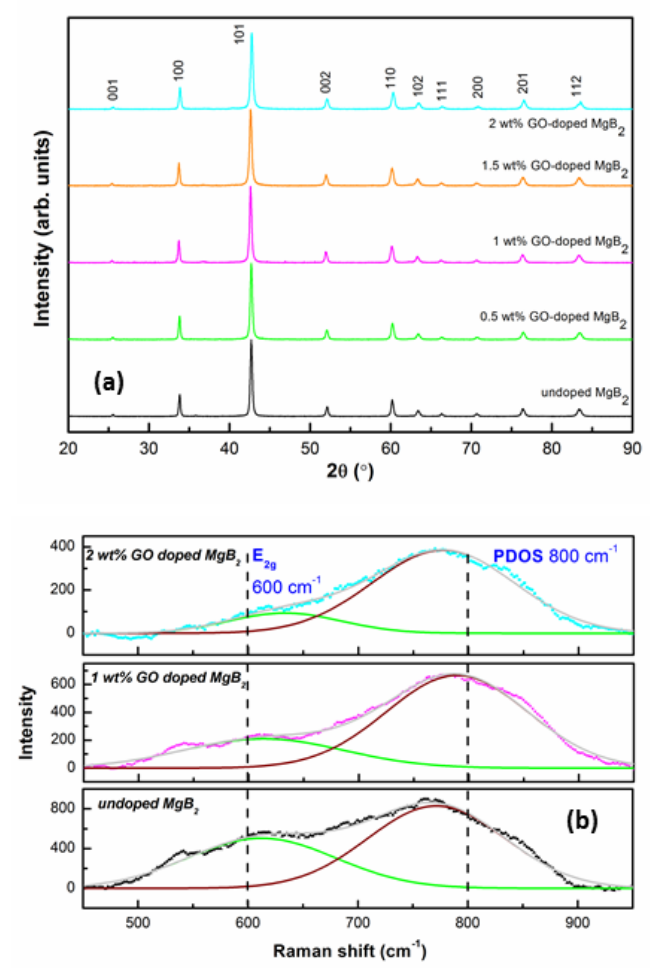

Figure 2.(a) XRD patterns for undoped and GO- doped $\mathrm{MgB}_{2}$.(b) Raman spectra with Gaussian fitted $\mathrm{E}_{2 \mathrm{~g}}$ mode and PDOS distortion of undoped GO-doped $\mathrm{MgB}_{2}$. 


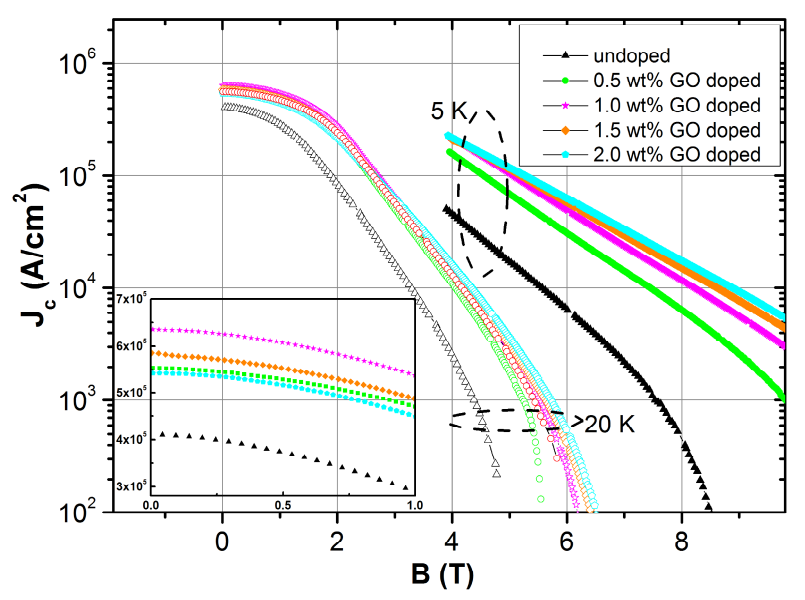

Figure 3. In-field $\mathrm{J}_{\mathrm{c}}$ performance of undoped, and GO-doped $\mathrm{MgB}_{2}$ bulk samples. Inset shows an larged view of the low-field $\mathrm{J}_{\mathrm{c}}$ at $20 \mathrm{~K}$ 